\title{
The efficacy and safety of posterior pole buckles in the control of progressive high myopia
}

\begin{abstract}
Purpose Degenerative myopia is a significant cause of vision loss; yet there is no accepted way of controlling its causative phenotype - progressive high axial myopia. Scleral reinforcement, introduced over 50 years ago, was discredited as a useful technique. This 5-year 'proof of concept' study examines buckling of the posterior pole for myopia control and follows the course of untreated fellow eyes.

Method A total of 59 adult eyes, with myopic refractive corrections ranging from -9 to $-22 \mathrm{D}$ and axial lengths from 27.8 to $34.6 \mathrm{~mm}$, were studied. A 1-cm-wide flexible buckle of donor sclera was positioned over the posterior pole and secured, under positive tension, to the anterior globe. The eyes were monitored for 5 years, as were unsupported fellow eyes. The axial lengths, visual acuities, and optical coherence tomography macular scans were collected and all complications were noted.

Results Over 5 years, axial length control was achieved by scleral buckling, whereas axial extension progressed in the untreated group. No serious complication occurred and no eye lost visual acuity from the procedure.

Temporary intra-ocular pressure elevation, small choroidal effusions, and variable periods of abduction limitation occurred after surgery. In one case of tractional myopic macular schisis, a full correction was achieved by buckling and visual acuity improved.

Eye (2009) 23, 2169-2174; doi:10.1038/eye.2008.433; published online 20 February 2009
\end{abstract}

Keywords: degenerative myopia; myopia; progressive myopia; macular traction; macular buckle; macular schisis

B Ward ${ }^{1}$, EP Tarutta ${ }^{2}$ and MJ Mayer ${ }^{3}$

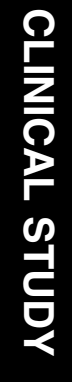

\section{Introduction}

Genetic factors are responsible for the majority of the cases of degenerative myopia. ${ }^{1}$ In addition, environmental and life-style factors have secondary developmental influences. ${ }^{2}$ In such eyes, the growth of the scleral shell is accelerated during childhood and adolescence, with further incremental increases in its dimensions occurring, by scleral stretching and thinning, in the adult years of life. The stretching of weak scleral collagen seems to result from internal and external applied forces, including the intra-ocular pressure. Progressive enlargement of the scleral shell creates shearing forces within the contiguous structures: the choroid, the retina, and the vitreous body. The failure of these tissues has serious effects on macular functioning and can also lead to retinal tears and detachment.

The increased recognition of myopia as a world health issue has brought new urgency to the need to understand the mechanisms of its development and to give direction to the pursuit of effective remedies. ${ }^{3}$ Yet, today, there exists no medical or surgical treatment that is widely accepted to be able to prevent, or to control, myopic globe enlargement and degenerative myopia progression.

A generation ago, surgical treatments were felt to hold the promise of axial myopia control. Surgeons sought to prevent axial elongation and staphyloma progression by the placement of grafts over the posterior part of the eye.

Reinforcement of the sclera was intended to limit both its growth and its stretching. Shevelev ${ }^{4}$ first proposed the transplantation of fascia lata for scleral reinforcement in 1930. Fifty years ago, Borley and Snyder ${ }^{5}$ described a technique for the placement of grafts of donor sclera. Curtin ${ }^{6}$ also advocated donor-sclera
${ }^{1}$ Retinal Diagnostic Center, 3803 S. Bascom Ave, no. 104, Campbell, CA, USA

${ }^{2}$ Helmholtz Institute for Research in Eye Diseases, Department of Myopia and Strabismus, Moscow, Russian Republic

${ }^{3}$ Department of Experimental Psychology, University of California, Santa Cruz, CA, USA

Correspondence: B Ward, Vitreoretinal Diseases, Retinal Diagnostic Center, 3803 S. Bascom Ave, no. 104, Campbell, CA 95008, USA

Tel: + (408) 559 6116; Fax: + (408) 3770811.

E-mail: bwardrdc@ yahoo.com

Received: 18 May 2008 Accepted in revised form: 22 December 2008 Published online: 20 February 2009

The material of this paper was presented at the Congress of the Royal College of

Ophthalmologists, Liverpool, England, May 2008.

All applicable institutional and governmental regulations were followed during this study.

Support was received from the Retinal Diagnostic Center Research Foundation of Campbell, CA, USA. Proprietory interest: None 
grafting for posterior ocular reinforcement. Momose ${ }^{7}$ introduced Lyodura, derived from processed cadaver dura mater, for scleral reinforcement in 1976. Later, Snyder and Thompson ${ }^{8}$ published accounts of their experiences with a modified scleral reinforcement technique. Thompson ${ }^{9}$ offered a further simplification of Borley and Snyder's scleral reinforcement approach in 1978.

After years of experience with their own variations of scleral reinforcement, both Thompson ${ }^{10}$ and Pruett ${ }^{11}$ expressed satisfaction with the efficacy and safety of their series of cases. In contrast, Curtin and Whitmore ${ }^{12}$ had negative conclusions on the outcomes for their reinforcement techniques. With no convincing proof of the safety or efficacy of scleral reinforcement, this approach became discredited and is largely un-used today.

Finding that vision loss, from myopic macular degeneration in later life, tended to correlate with an eye's axial length, it seemed that axial control remained a worthwhile treatment goal. ${ }^{13,14}$ Reviewing the literature of scleral grafting, it appeared that the support had been too little, and that it had been applied too late in the course of the pathological process. The application of stronger positive forces to the back of the eye, such as from a tensioned flexible buckle, was reasoned to be likely to give superior restraint to axial elongation. It was felt that Laplace's law, modelling the factors affecting tension in the wall of a fluid-filled vessel, provided support to such a proposition. The equation $T=\left(P-P^{\prime}\right) R / M$ predicts wall tension $(T)$ to be reduced by decreasing the trans-mural pressure gradient $\left(P-P^{\prime}\right)$ and the radius of curvature of the wall $R$, and by increasing the wall's thickness $M$. Tensioned scleral buckles may be reasoned to be capable of reducing both $\left(P-P^{\prime}\right)$ and $R$, while increasing $M$.

\section{Materials, methods, and ethics}

A total of 59 patients constitute the subjects of this retrospective clinical study. The buckled (treated) and control (untreated) eyes had axial lengths ranging from 27.8 to $34.6 \mathrm{~mm}$, and myopic refractive errors ranging from -9.00 to $-22.00 \mathrm{D}$. All patients were offered scleral buckling because they had ocular axial lengths in excess of $27.0 \mathrm{~mm}$ and evidence of continuing axial myopia progression. All eyes showed some degree of myopic macular degeneration. The visual acuities ranged from $20 / 25$ to $6 / 400$.

Informed consent was obtained after a full discussion of both the possible complications and the desired positive outcomes. All institutional and governmental regulations were followed in this study. The patients were made aware of the fact that their axial length measurements had been found to be over the threshold of significant risk for future myopic macular degeneration and visual acuity loss. The goal of the procedure was stated to be the constraint of further axial myopia progression, in the hope of limiting future macular degeneration. It was not found possible to randomize the eye to be treated in the majority of patients. Patients were requested to allow a control eye to be followed for at least 2 years. The data from eyes which had had buckling surgery for retinal detachment repair, uncontrolled glaucoma, or any other non-myopia-related vision-threatening condition were excluded from the study. Paediatric cases were also excluded, the age range of the patients being from 18 to 68 years with an average of 39 years.

Before surgery, both eyes underwent a baseline examination with measurements of refractive errors, visual acuities, intra-ocular pressures, a full ocular assessment, optical coherence tomography of the macula, and axial length measurement by A-mode ultrasonography and IOLMASTER ${ }^{\circledR}$ reflected light biometry. Thus, complete pre-operative baseline data were also available for the un-operated 'fellow' eye.

\section{Surgical technique}

The procedure was carried out under general anaesthesia, to avoid the creation of orbital oedema by the injection of anaesthetic fluids. The buckling elements in this series of cases were cut from the sclera and cornea of donor eyes, supplied by the regional eye bank. These eyes had been tested and certified to be free of potential pathogens, according to the bank's approved testing protocols. The material was fashioned into strips, 70-80 $\mathrm{mm}$ long and $10-12 \mathrm{~mm}$ wide in their central parts. They were shaped to facilitate positioning over the back of the eye, and to rest nasal to the inferior oblique muscle insertion and temporal to the optic nerve, without the need for exposure or suturing at the posterior part of the globe. Figure 1 is a schematic representation of a buckle secured over the posterior pole of a right eye. The buckle's tendency to slip temporally was constrained
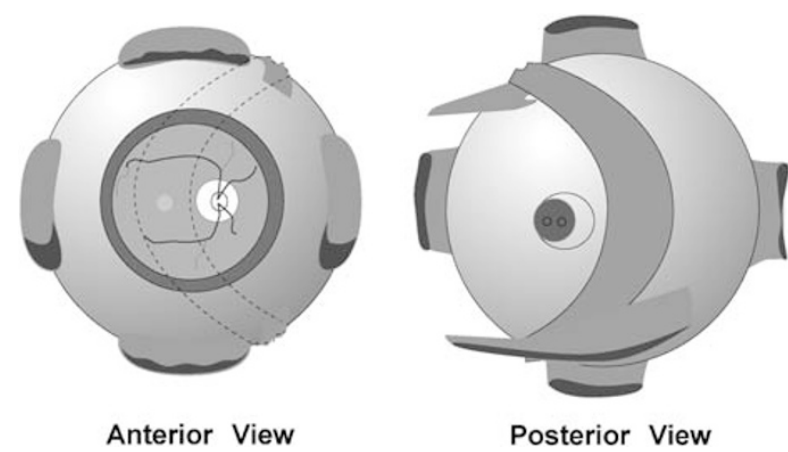

Figure 1 Schematics of the anterior and posterior aspects of a right eye, illustrating the positioning of a tensioned posterior polar buckle. 
partly by the nasal portion of the inferior oblique muscle's insertion, but most importantly by the selection of the points at which its ends were sutured to the anterior sclera in the nasal quadrants of the globe. After placement, the buckle's position could be judged by direct observations, with local tissue retraction by means of an Arruga spoon.

The surgical approach begins with a limbal peritomy. Silk ties are passed under each of the four rectus muscles and the inferior oblique. The inter-muscular septi and adhesions between the muscles and the globe are divided to allow passage of the buckle.

The buckling material is first placed under the superior rectus muscle, and the nasal end is secured to the sclera in the supero-nasal quadrant with two 5-0 Dacron ${ }^{\mathbb{R}}$ sutures, placed just posterior to the line of insertion of the rectus muscles.

The temporal end of the element is then directed inferiorly, to pass medial to the lateral rectus muscle, behind the insertion of the inferior oblique and between the inferior rectus muscle and the globe, so that the free end enters the infero-nasal quadrant. Holding the free end in straight forceps, a series of circular vertical movements is made, with variable tension applied to the strip. This movement causes the central portion to slide posteriorly over the temporal globe, to rest over the posterior pole. Once the element's position has been verified, the free end is cut to length and tensioned as more anterior-placed scleral sutures are tightened. Apical flattening of a posterior staphyloma may be seen during the routine ophthalmoscopic check of central retinal artery perfusion.

\section{Post-surgical monitoring}

After surgery, a topical steroid/antibiotic combination drop is used four times daily, and tapered off over 3 weeks. A topical pressure-reducing agent is required for a period of up to 2 weeks. The pressure-controlling agent is discontinued when the intra-ocular pressure measures no more than $17 \mathrm{~mm} \mathrm{Hg}$, without medication.

The eyes are re-evaluated at the third month, and thereafter at intervals of 6-9 months. The data and observations used in this report were collected by a number of experienced clinicians who had no knowledge of this clinical study.

\section{Results}

\section{The complications}

The complications in this series of 59 adult scleral buckles were found to be those that are familiar from scleral buckling for retinal detachment repair. In descending order of incidence, these were: temporary abduction weakness (59/59), temporary intra-ocular pressure elevation $(4)^{*}$, shallow choroidal effusions $(11 / 59)$, and motility restriction in elevation (2/59). The asterisk $\left.{ }^{*}\right)$ indicates the fact that after the fourth case, pressure-control medication was begun at the end of all procedures. The temporary elevation of the intra-ocular pressure occurred in virtually all eyes. The temporary post-operative abduction deficiency was responsible for the most common post-operative patient complaint: a binocular diplopia in lateral gaze to the side of the operated eye. Rare cases had symptoms of lateral rectus weakness for more than 2 months. One eye required the surgical relief of a limitation to up-gaze, caused by the tethering effects of scar tissue involving the insertions of the inferior rectus and medial rectus muscles.

\section{Axial myopia control}

The yearly data for the mean values and the standard deviations of the axial length changes, from preoperative baseline levels, are presented in Figure 2. At the time of the surgery on the first eye, 29 patients had had a fellow eye similar enough to the buckled one to be designated as an untreated control.

The data show that the procedure was effective in limiting axial extension over the 5-year study period. By year 5, the control group contained insufficient eyes for statistical validity. The unsupported eyes had an average axial elongation of $0.6 \mathrm{~mm}$ over the first 3 years of the study, equivalent to an increase in axial myopia of almost 2 D. This difference in axial extension between the groups is made more significant by the fact that the treated eyes were not randomized, and the eye to be supported was designated by 'patient's choice'. That choice most commonly meant that the control eye was in fact the one with the better acuity, the lower degree of myopia, or the lower historical rate of axial myopia progression.

In Figure 3, the mean visual acuities of the groups of eyes do not satisfy statistical tests for the reliability of differences of the means. On the other hand, individual patients in whom a meaningful visual acuity improvement did occur were identified, and in one case this improvement was substantial. Figures 4 and 5 are optical coherence tomography studies (identical vertically oriented cuts) involving the macula of a 68-year-old pseudo-phakic white female (visual acuity 20/80 and axial length $30.0 \mathrm{~mm}$ ) who experienced a visual acuity increase to $20 / 40$, by 4 months after posterior pole buckling. The images show the relief of myopic vitreo-macular traction, associated macular schisis, and retinal detachments. In addition to increased clarity of vision, the patient reported increased 


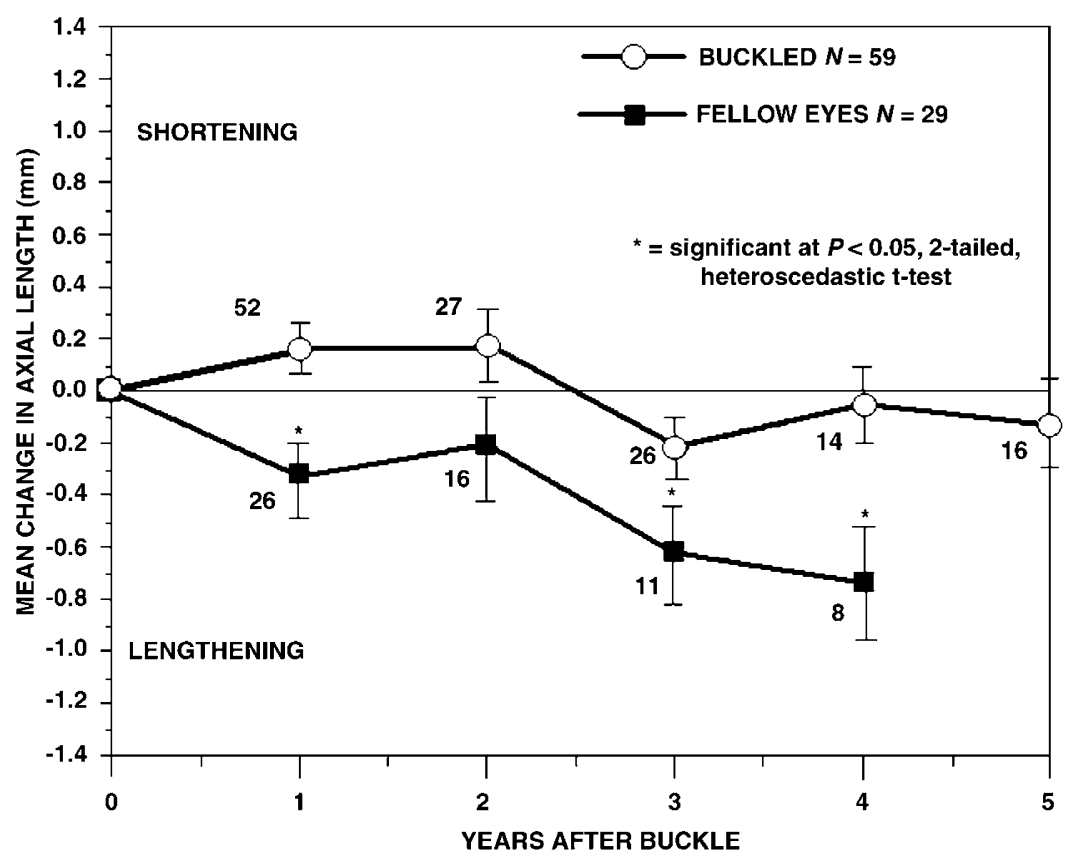

Figure 2 Five-year data of average axial length changes after scleral buckling and 4-year data for the untreated fellow eyes. The standard deviations of the means are shown for each data point. The horizontal line at $0 \mathrm{~mm}$ indicates no change in axial length from pre-operative levels. Values above the line indicate decreases in length, while increases are plotted below it.

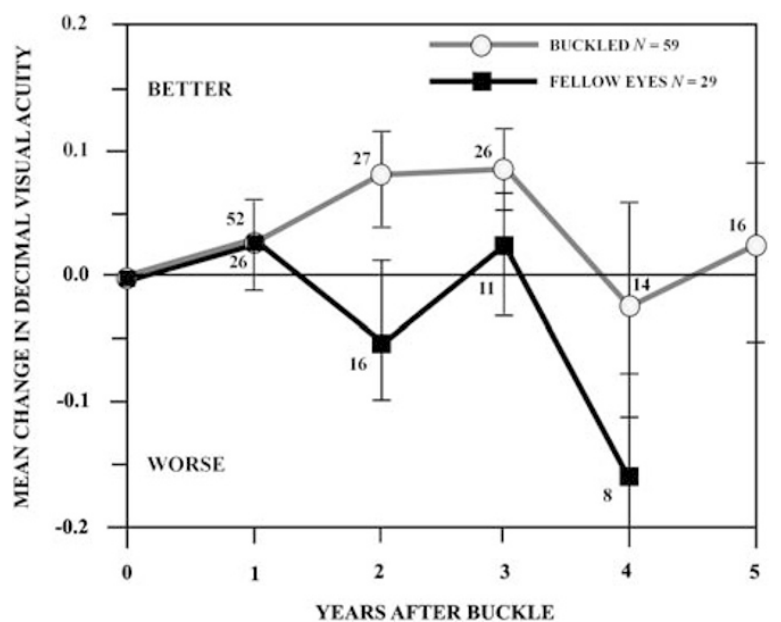

Figure 3 Five-year follow-up data of the means of the average fractional visual acuity changes, from the pre-operative values, in buckled eyes and their untreated fellow eyes. The standard deviations of the mean are indicated at each data point. The horizontal line at 0 represents no visual acuity change. Improvements of acuity are recorded above the line, while reductions are plotted below it.

brightness and colour saturation, and a reduction in the distortion of straight lines.

All eyes in this study had atrophic myopic macular degeneration, with RPE de-pigmentation and pigment clumping, as well as choroidal thinning. Macular haemorrhage from choroidal vessel incursion was seen in three treated eyes and two control eyes during the course of the study. Myopic macular schisis was present in one eye, and visual acuities were also affected by changes in nuclear sclerosis of the lens.

\section{Discussion}

Adult eyes with high axial myopia may show increments of axial extension at any age, as reflected in the data for the un-treated eyes in this study. Any extension increases the risk of macular degeneration later in life. Axial length stability was found in this non-randomized study of 59 treated eyes, whereas axial progression occurred in the fellow eyes. The two-tailed heteroscedastic $t$-test showed the validity of differences of the means of the two groups. Some patients were lost to follow-up, and a number of patients requested that the procedure be carried out on the second eye. This attrition of the control group limited the statistical validity of their data to a period of 4 years.

The absence of any vision-threatening complication was a positive outcome of this study. The most common complications were temporary: a lateral rectus weakness, post-operative intra-ocular pressure increase, and small choroidal effusions. The muscle weakness is likely to result from muscle denervation or ischaemia and orbital scar-formation. The early intra-ocular pressure increases and small choroidal effusions are both likely to be 


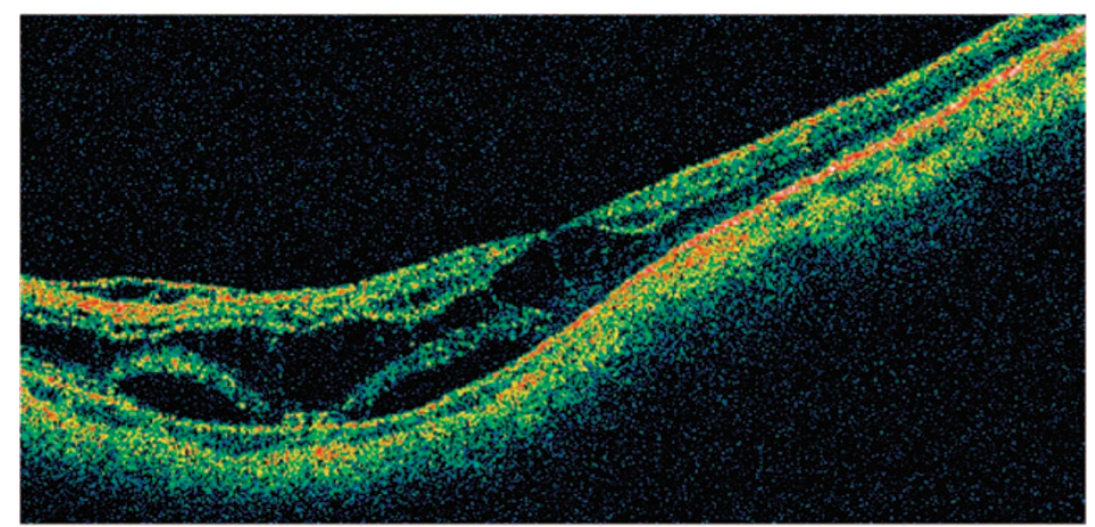

Figure 4 The optical coherence tomogram (OCT) of the left macula of a 69-year-old pseudo-phakic female. The eye had an axial length of $30.0 \mathrm{~mm}$, tractional retinal schisis, and detachment. This image shows the macula before posterior pole buckling.

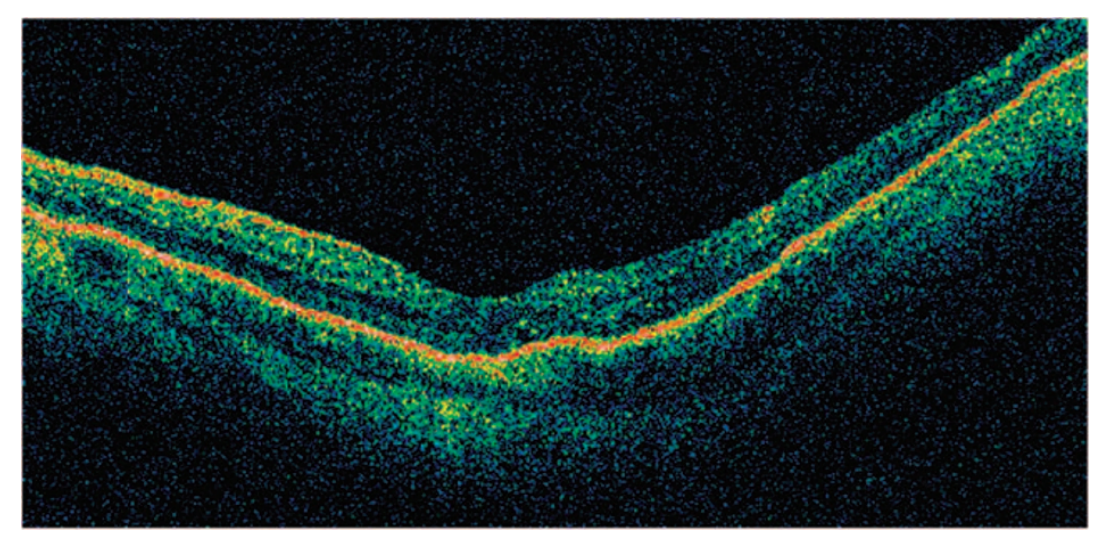

Figure 5 The OCT of the eye shown in Figure 4, with the identical vertical section orientation on the day after a tensioned scleral buckle had been applied to the posterior pole.

associated with the buckle's compression of vortex veins and other fluid-egress channels. Pre-operative counselling allowed the patients to be prepared for an uncertain period of diplopia in lateral gaze (to the side of the operated eye), and created awareness of the need for a period of topical medication for intra-ocular pressure control.

The data support the proposition that axial myopia progression may be limited by posterior pole buckling. The control of axial myopia progression provides the hope of minimizing myopic macular degeneration, a topic requiring study over longer periods of time. Myopia control offers other benefits, including the extension of the useful life of optical corrections, including those provided by various forms of refractive surgery.

The incidental single finding that a buckle relieved the neural disruptive effects of myopic macular traction suggests a treatment option for this condition. Whereas vitrectomy and pre-retinal membrane peeling release myopic macular traction, ${ }^{14}$ buckling is an approach that avoids intra-ocular surgery, with the additional benefit of axial myopia control.

In conclusion, the experience with 59 cases showed effective axial myopia control and an acceptable safety profile for posterior pole buckling. No case of visual acuity loss occurred with the procedure. Three common temporary complications were: intra-ocular pressure elevation, a period of under-action of the lateral rectus muscle, and small choroidal effusions. One case with myopic macular schisis was corrected by buckling. The effects of buckling on the progression of myopic macular degeneration and visual acuity require prospective study over longer periods of time.

\section{References}

1 Curtin BJ. The nature of pathological myopia. In: The Myopias. Harper \& Row: Philadelphia, 1985; 237-239.

2 Saw SM, Chua WH, Wu HM, Yap E, Chia KS, Stone RA. Myopia gene-environment interaction. Ann Acad Med Singapore 2000; 29(3): 290-297. 
3 Tano Y. Pathological myopia, where are we now? Am J Ophthalmol 2002; 134: 645-660.

4 Shevelev MM. Operation against high myopia and scleralectasia with aid of the transplantation of fascia lata on thinned sclera. Russian Oftalmol J 1930; 11(1): 107-110.

5 Borley WE, Snyder AA. Surgical treatment of high myopia: scleral reinforcement. Trans Am Acad Ophthalmol Otolaryngol 1958; 62: 791-802.

6 Curtin BJ. Surgical support of the posterior sclera: Part II. Clinical results. Am J Ophthalmol 1961; 52: 253.

7 Momose A. Surgical treatment of Myopia - with special references to posterior scleral support operation and raclial keratotomy. Indian J Ophthal 1983; 31: 759-767.

8 Snyder AA, Thompson FB. A simplified technique for surgical treatment of degenerative myopia. Am J Ophthalmol 1972; 74: 273-277.
9 Thompson FB. A simplified scleral reinforcement technique. Am J Ophthalmol 1978; 86: 782-790.

10 Thompson FB. Scleral Reinforcement. Chapter 10. Myopia Surgery. Macmillan: New York, 1990, pp 267-297.

11 Pruett RP. Myopia (Posterior Segment) Chapter 121 in Master Techniques in Ophthalmic Surgery. Williams \& Wilkins: Baltimore, 1995.

12 Curtin BJ, Whitmore WG. Long-term results of scleral reinforcement. Am J Ophthalmol 1987; 103: 544-548.

13 Curtin BJ, Karlin DB. Axial length measurements and fundus changes in the myopic eye. Am J Ophthalmol 1971; 71: 42-53.

14 Takano M, Kishi S. Foveal retinoschisis and retinal detachment in severely myopic eyes with posterior staphyloma. Am J Ophthalmol 1999; 128: 472-476. 